\title{
Strong coupling constant and four-quark condensates from vacuum polarization functions with dynamical overlap fermions
}

\author{
Eigo Shintani ${ }^{* 1}$, S. Aoki ${ }^{2,3}$, S. Hashimoto ${ }^{1,4}$, H. Matsufuru ${ }^{1}$, J. Noaki ${ }^{1}$, T. Onogi $^{5}, \mathbf{N}$. \\ Yamada $^{1,4}$ (for JLQCD Collaboration) \\ ${ }^{1}$ High Energy Accelerator Research Organization (KEK), Tsukuba 305-0801, Japan \\ ${ }^{2}$ Graduate School of Pure and Applied Sciences, University of Tsukuba, Tsukuba 305-8571, \\ Japan, \\ ${ }^{3}$ Riken BNL Research Center, Brookhaven National Laboratory, Upton, NY 11973, USA, \\ ${ }^{4}$ School of High Energy Accelerator Science, The Graduate University for Advanced Studies \\ (Sokendai), Tsukuba 305-0801, Japan, \\ ${ }^{5}$ Yukawa Institute for Theoretical Physics, Kyoto University, Kyoto 606-8502, Japan
}

\begin{abstract}
We study the vacuum polarization functions on the lattice with exact chiral symmetry of overlap fermion by matching the lattice data at large momentum scales with the Operator Product Expansion (OPE). We extract the strong coupling constant $\alpha_{s}(\mu)$ in two-flavor QCD as $\Lambda \frac{(2)}{M S}=$ $0.234(9)\left(\begin{array}{c}+16 \\ -0\end{array}\right) \mathrm{GeV}$. From the analysis of the difference between the vector and axial-vector channels, we extract some of the four-quark (dimension-six) condensates.
\end{abstract}

The XXVI International Symposium on Lattice Field Theory

July 14 - 19, 2008

Williamsburg, Virginia, USA

\footnotetext{
* Speaker.

†shintani@post.kek.jp
} 


\section{Introduction}

In Quantum Chromodynamics (QCD) the vacuum polarization, defined through the (axial)vector current correlator, contains rich information of its perturbative and non-perturbative dynamics. In the long distance regime it is sensitive to the low-lying particle spectrum. The short distance regime, on the other hand, can be analyzed using perturbation theory supplemented by the Operator Product Expansion(OPE). The current correlator can be expressed as an expansion in terms of the strong coupling constant $\alpha_{s}$ together with power corrections of the form $\left\langle\mathscr{O}^{(n)}\right\rangle / Q^{n}$. Here, the local operator $\mathscr{O}^{(n)}$ has a mass dimension $n$ and $Q$ is the momentum scale flowing into the correlator. If one can calculate the correlators non-perturbatively, theoretical determination of those fundamental parameters is made possible.

Lattice QCD calculation offers such a non-perturbative technique. Two-point correlators can be calculated for space-like separations. In this work we investigate the use of the perturbative formulae of the correlators for the lattice data obtained in the high $Q^{2}$ regime. By inspecting the numerical data, we find that this is indeed possible at a lattice spacing $a \simeq 0.12 \mathrm{fm}$ if we subtract the bulk of the discretization effects non-perturbatively. The remaining effect can be estimated using the perturbation theory. To our knowledge quantitative analysis including the determination of $\alpha_{s}$ and $\left\langle\mathscr{O}^{(n)}\right\rangle$ has been missing until recently.

\section{Vacuum polarization function and $\mathrm{OPE}$}

While the vacuum polarizations $\Pi_{J}\left(Q^{2}\right)(J$ denotes vector or axial-vector channel) are ultraviolet divergent and their precise value depends on the renormalization scheme, their derivative $D_{J}\left(Q^{2}\right)=-Q^{2} d \Pi_{J}\left(Q^{2}\right) / d Q^{2}$, called the Adler function [1], is finite and renormalization scheme independent. Therefore, the continuum perturbative expansion of $D_{J}\left(Q^{2}\right)$ to order $\alpha_{s}^{3}$ [2], can be directly applied to the lattice data. They include the parameters describing the gluon condensate $\left\langle\alpha_{s} G^{2}\right\rangle / Q^{4}$, the quark condensate $\langle m \bar{q} q\rangle / Q^{4}$, and four-quark condensates $\left\langle O_{8}\right\rangle / Q^{6}$ and $\left\langle O_{1}\right\rangle / Q^{6}$. (The explicit forms of $O_{8}$ and $O_{1}$ are given in [3].)

In the continuum theory, the vacuum polarization functions $\Pi_{J}^{(\ell)}\left(Q^{2}\right)$ are defined through

$$
\begin{aligned}
& \left\langle J_{\mu} J_{v}\right\rangle(Q) \equiv \int d^{4} x e^{i Q \cdot x}\left\langle T\left\{J_{\mu}^{i j}(x) J_{v}^{j i}(0)\right\}\right\rangle \\
& =\left(\delta_{\mu v} Q^{2}-Q_{\mu} Q_{v}\right) \Pi_{J}^{(1)}\left(Q^{2}\right)-Q_{\mu} Q_{v} \Pi_{J}^{(0)}\left(Q^{2}\right)
\end{aligned}
$$

where the current $J_{\mu}^{i j}$ may either be a vector current $V_{\mu}^{i j}=\bar{q}_{i} \gamma_{\mu} q_{j}$ or an axial-vector current $A_{\mu}^{i j}=$ $\bar{q}_{i} \gamma_{\mu} \gamma_{5} q_{j}$ with flavor indices $i \neq j . \Pi_{J}^{(1)}\left(Q^{2}\right)$ and $\Pi_{J}^{(0)}\left(Q^{2}\right)$ denote the transverse and longitudinal parts of the vacuum polarization, respectively. For the vector channel $(J=V), \Pi_{V}^{(0)}\left(Q^{2}\right)=0$ is satisfied due to current conservation. For the axial-vector channel $(J=A)$, the longitudinal component may appear when the quark mass is finite.

With the overlap fermion, we use the currents $V_{\mu}^{i j}=Z_{V} \bar{q}_{i} \gamma_{\mu}\left(1-D / 2 m_{0}\right) q_{j}$ and $A_{\mu}^{i j}=Z_{A} \bar{q}_{i} \gamma_{\mu} \gamma_{5}(1-$ $\left.D / 2 m_{0}\right) q_{j}$, which are not conserving but form a multiplet of the axial transformation as they do in the continuum theory. $D$ denotes the massless overlap-Dirac operator, and the parameter $m_{0}$ is fixed at 1.6. The renormalization factor $Z \equiv Z_{V}=Z_{A}$ has been calculated non-perturbatively, $Z=$ 
1.3842(3) [4]. Taking account of remaining symmetries on the lattice (parity and cubic symmetries), the correlators of these lattice currents can be expressed as

$$
\begin{aligned}
& \left\langle J_{\mu} J_{v}\right\rangle^{\text {lat }}(Q)=\Pi_{J}^{(1)}(Q) Q^{2} \delta_{\mu v}-\Pi_{J}^{(0+1)}(Q) Q_{\mu} Q_{v} \\
& -\sum_{n=0}^{\infty} B_{n}^{J}(Q) Q_{\mu}^{2 n} \delta_{\mu v}-\sum_{m, n=1}^{\infty} C_{m n}^{J}(Q)\left\{Q_{\mu}^{2 m+1} Q_{v}^{2 n-1}+Q_{v}^{2 m+1} Q_{\mu}^{2 n-1}\right\}
\end{aligned}
$$

where $\Pi_{J}^{(0+1)}(Q) \equiv \Pi_{J}^{(0)}(Q)+\Pi_{J}^{(1)}(Q)$. The lattice momentum $Q_{\mu}$ is defined as $Q_{\mu}=(2 / a) \sin \left(\pi n_{\mu} / L_{\mu}\right)$ with an integer four-vector $n_{\mu}$ whose components take values in $\left[0, L_{\mu} / 2\right]$ on a lattice of size $L_{\mu}$ in the $\mu$-th direction $\left(L_{i=1,2,3}=16\right.$ and $\left.L_{t}=32\right)$. On the lattice, $\Pi_{J}^{(\ell)}(Q)$ is not just a function of $Q^{2}$ but of $Q_{\mu}$ in general due to the violation of the Lorentz symmetry. In Ref.[5] we describe the detail of derivation of these contribution to vacuum polarization, $\Pi_{V+A}\left(Q^{2}\right)$ and $\Pi_{V-A}\left(Q^{2}\right)$.

We now discuss the fit of the lattice data to the OPE expression of the form [6]

$$
\begin{aligned}
\left.\Pi_{J}^{(0+1)}\right|_{\mathrm{OPE}}\left(Q^{2}\right) & =c+C_{0}\left(Q^{2}, \mu^{2}\right)+\frac{C_{m}^{J}\left(Q^{2}\right)}{Q^{2}}+C_{\bar{q} q}^{J}\left(Q^{2}\right) \frac{\langle m \bar{q} q\rangle}{Q^{4}} \\
& +C_{G G}\left(Q^{2}\right) \frac{\left\langle\left(\alpha_{s} / \pi\right) G G\right\rangle}{Q^{4}} .
\end{aligned}
$$

Instead of directly treating the Adler function, we analyze its indefinite integral $\left.\Pi_{J}^{(0+1)}\right|_{\mathrm{OPE}}\left(Q^{2}\right)$. A constant $c$ is scheme-dependent, while other terms are finite and well defined. The leading term, $C_{0}\left(Q^{2}, \mu^{2}\right)$, is known to $\mathscr{O}\left(\alpha_{s}^{2}\right)$ in the massless limit [2]. For a finite quark mass there is a contribution of $\mathscr{O}\left(m^{2} / Q^{2}\right)$, which is represented by the term $C_{m}^{J}\left(Q^{2}\right)$ with running mass $m=m(\mu)$ at scale $\mu$, known to $\mathscr{O}\left(\alpha_{s}^{2}\right)$. We ignore terms of $\mathscr{O}\left(m^{4}\right)$ and higher. The OPE corrections of the form $\left\langle O^{(n)}\right\rangle / Q^{n}$ start from the dimension-four operators $m \bar{q} q$ and $\left(\alpha_{s} / \pi\right) G G$. Their Wilson coefficients $C_{\bar{q} q}^{J}\left(Q^{2}\right)$ and $C_{G G}\left(Q^{2}\right)$ are known to $\mathscr{O}\left(\alpha_{s}^{2}\right)$ [7]. The terms of order $1 / Q^{6}$ and higher are not included. The perturbative expansions are consistently given in terms of the strong coupling constant $\alpha_{s}(\mu)$ defined in the $\overline{\mathrm{MS}}$ scheme.

Here we note that the "gluon condensate" $\left\langle\left(\alpha_{s} / \pi\right) G G\right\rangle$ is defined only through the perturbative expression like (2.3). Due to an operator mixing with the identity operator, the operator $\left(\alpha_{s} / \pi\right) G G$ contains a quartic power divergence that cannot be unambiguously subtracted within perturbation theory, which is known as the renormalon ambiguity [8]. Therefore, the term $\left\langle\left(\alpha_{s} / \pi\right) G G\right\rangle$ in (2.3) only has a meaning of a parameter in OPE, that may depend on the order of the perturbative expansion, for instance. The quark condensate $\langle\bar{q} q\rangle$ is well-defined in the massless limit, since it does not mix with lower dimensional operators, provided that the chiral symmetry is preserved on the lattice. Power divergence may appear at finite quark mass as $m a^{-2}$. In the OPE formula (2.3), it thus leads to a functional dependence $m^{2} a^{-2} / Q^{4}$. In our numerical analysis we neglect this quadratic term in $m$, as it should be smaller than the already small leading $m$ dependence from the quark condensate.

In addition to the individual vector and axial-vector correlators, we consider the $V-A$ vacuum polarization function. For the difference $\Pi_{V-A}^{(0+1)}(Q) \equiv \Pi_{V}^{(0+1)}(Q)-\Pi_{A}^{(0+1)}(Q)$, the lattice data are more precise than the individual $\Pi_{J}^{(0+1)}(Q)$, so that the $1 / Q^{6}$ and $1 / Q^{8}$ terms are also necessary:

$$
\left.\Pi_{V-A}^{(0+1)}\right|_{\mathrm{OPE}}\left(Q^{2}\right)=\left(C_{m}^{V}-C_{m}^{A}\right)\left(Q^{2}\right) \frac{1}{Q^{2}}+\left(C_{\bar{q} q}^{V}-C_{\bar{q} q}^{A}\right)\left(Q^{2}\right) \frac{\langle m \bar{q} q\rangle}{Q^{4}}
$$




$$
+\left(a_{6}(\mu)+b_{6}(\mu) \ln \frac{Q^{2}}{\mu^{2}}+c_{6} m_{q}\right) \frac{1}{Q^{6}}+\frac{a_{8}}{Q^{8}} .
$$

In the $V-A$ combinations the coefficients $C_{m}^{V}-C_{m}^{A}$ and $C_{\bar{q} q}^{V}-C_{\bar{q} q}^{A}$ start at $\mathscr{O}\left(\alpha_{s}\right)$. The dimension-six operators $a_{6}(\mu)$ and $b_{6}(\mu)$ contain the expectation values of dimension-six operators $O_{8}$ and $O_{1}$ [3]. The scale $\mu$ is set to $2 \mathrm{GeV}$. Unlike the dimension-four quark condensate $\langle m \bar{q} q\rangle,\left\langle O_{8}\right\rangle$ and $\left\langle O_{1}\right\rangle$ remain finite in the massless limit, hence gives leading contribution. The term $c_{6}$, which has a mass-dimension five, describes their dependence on the quark mass. The term $a_{8} / Q^{8}$ represents the contributions from dimension eight operators.

\section{Numerical results}

We use the lattice data from two-flavor QCD simulation with dynamical overlap fermions [9]. The simulations are performed at lattice spacing $a=0.118(2) \mathrm{fm}$ on a $16^{3} \times 32$ lattice, corresponding to the physical volume $(1.9 \mathrm{fm})^{3}$. The quark masses $m_{q}$ in this analysis are $0.015,0.025,0.035$ and 0.050 in the lattice unit, that cover the range $\left[m_{s} / 6, m_{s} / 2\right]$ with $m_{s}$ the physical strange quark mass. The main advantage of this data set is that both the sea and valence quarks preserve exact chiral and flavor symmetries by the use of the overlap fermion formulation [10]. The perturbative formulae for the vacuum polarizations can therefore be applied without any modification due to explicit violation of the chiral symmetry.

In the fitting of the lattice data with the functions (2.3) and (2.4), we use the value of the quark condensate obtained from a simulation in the $\varepsilon$-regime using the same lattice formulation at slightly smaller lattice spacing, $\langle\bar{q} q\rangle(2 \mathrm{GeV})=-[0.251(7)(11) \mathrm{GeV}]^{3}[11]$. The renormalization scale $\mu$ is set to $2 \mathrm{GeV}$. The quark mass is renormalized in the $\overline{\mathrm{MS}}$ scheme using the non-perturbative matching factor $Z_{m}(2 \mathrm{GeV})=0.838(17)$ [4] as $m(\mu)=Z_{m}(\mu) m_{q}$. The coupling constant $\alpha_{s}(\mu)$ is transformed to the scale of two-flavor QCD, $\Lambda \frac{(2)}{\mathrm{MS}}$, using the four-loop formula [12]. Then, the free parameters are the scheme-dependent constant $c,\left\langle\left(\alpha_{s} / \pi\right) G G\right\rangle$, and $\Lambda \frac{(2)}{\mathrm{MS}}$ for the fit of an average $\Pi_{V+A}^{(0+1)}(Q) \equiv \Pi_{V}^{(0+1)}(Q)+\Pi_{A}^{(0+1)}(Q)$. For the difference $\Pi_{V-A}^{(0+1)}(Q), \Lambda_{\overline{\mathrm{MS}}}^{(2)}$ obtained above is used as an input and the dimension-six condensates $a_{6}, b_{6}$ and $c_{6}$ are free parameters.

The OPE analysis requires a window in $Q^{2}$ where the systematic errors are under control. The upper limit $(a Q)_{\max }^{2} \simeq 1.324$ is set by taking the points where different definitions of the lattice momentum, i.e. $Q_{\mu}=(2 / a) \sin \left(\pi n_{\mu} / L_{\mu}\right)$ and $Q_{\mu}=(2 / a) \pi n_{\mu} / L_{\mu}$, give consistent results within one standard deviation. In the physical unit, this corresponds to $1.92 \mathrm{GeV}$. To determine $(a Q)_{\min }^{2}$, we investigate the dependence of the fit parameters on $(a Q)_{\min }^{2}$ in Figure 1. From the left three panels, we observe that the results for $\Lambda \frac{(2)}{\mathrm{MS}},\left\langle\left(\alpha_{s} / \pi\right) G G\right\rangle$, and $c$ are stable between $(a Q)_{\min }^{2} \simeq 0.48$ and 0.65 , which correspond to the momentum scale 1.16-1.35 GeV. Above $(a Q)_{\min }^{2} \simeq 0.65$ the fit becomes unstable; the results are still consistent within one standard deviation.

Similar plots (right panel) are shown for $a_{6}, b_{6}$ and $a_{8}$ obtained from the fit of $\Pi_{V-A}^{(0+1)}(Q)$. We attempt to fit with (filled symbols) and without (open symbols) the $a_{8} / Q^{8}$ term in order to investigate how stable the results are against the change of the order of the $1 / Q^{2}$ expansion. We find that the fit with $a_{8} / Q^{8}$ is stable down to $(a Q)_{\min }^{2} \simeq 0.46$, while the other could not be extended below $(a Q)_{\min }^{2} \simeq 0.58$. The difference between filled and open symbols is marginal for $a_{6}$ (circles), 

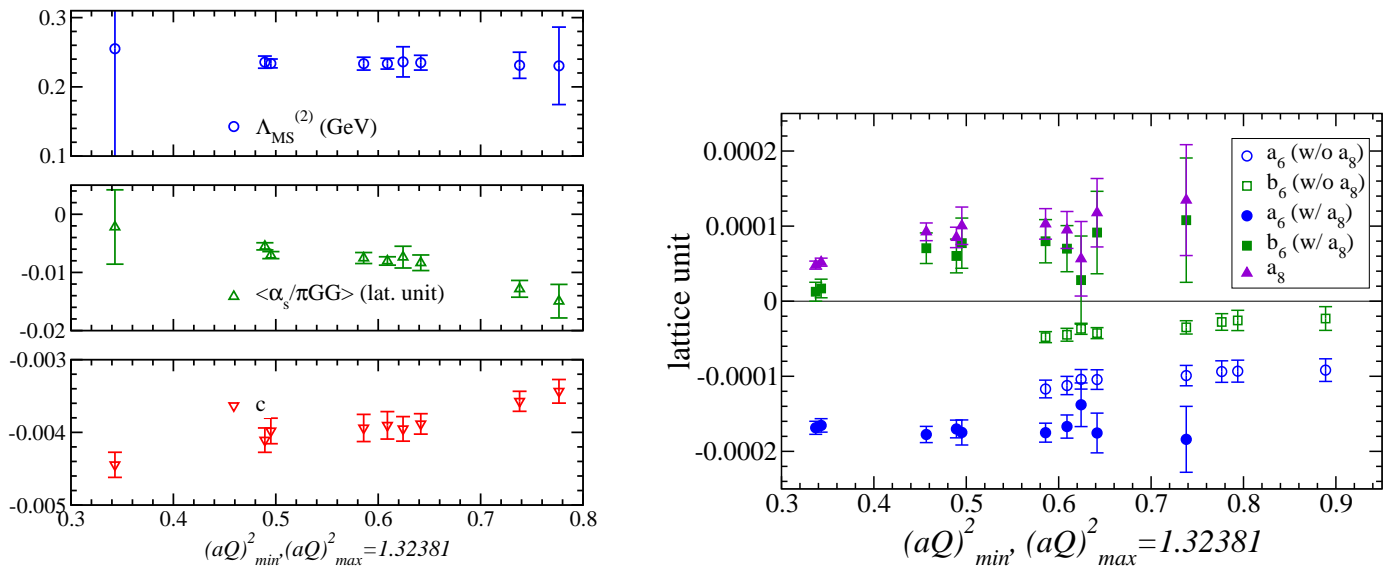

Figure 1: Fit range dependence of $\Lambda_{\overline{M S}}^{(2)},\left\langle\left(\alpha_{s} / \pi\right) G G\right\rangle$ and the constant term $c$ (left). Similar plots for $a_{6}(\mu)$, $b_{6}(\mu)$ and $a_{8}$ (right). The horizontal axis denotes the minimum momentum squared $(a Q)_{\min }^{2}$.
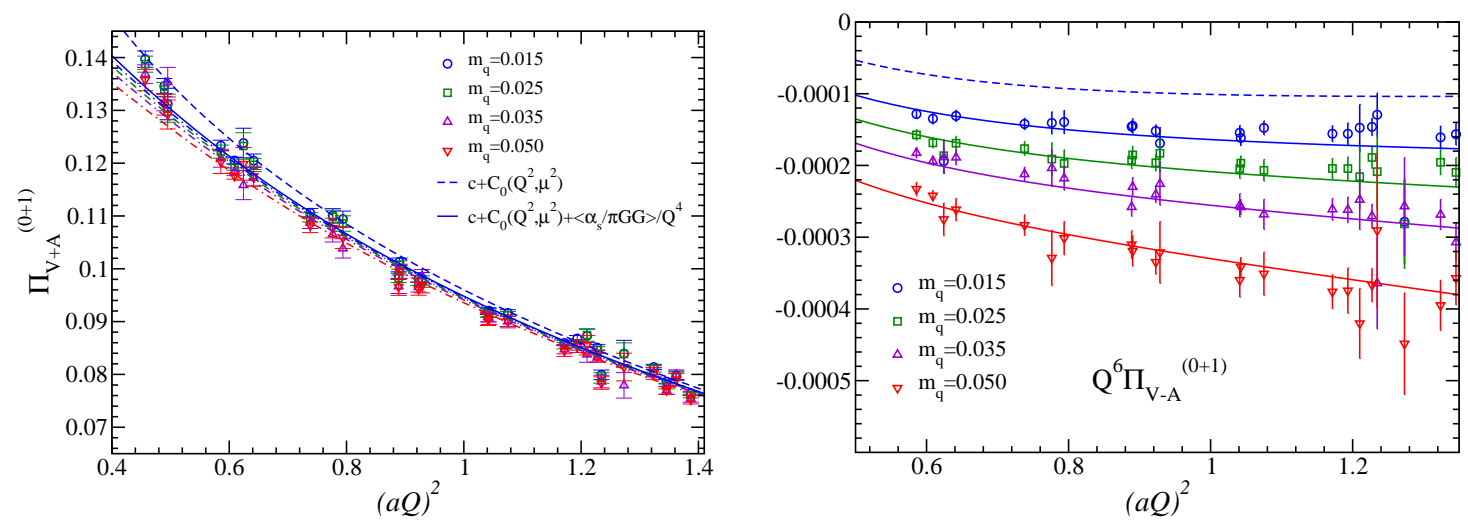

Figure 2: $\Pi_{V+A}^{(0+1)}(Q)$ (left panel) and $Q^{6} \Pi_{V-A}^{(0+1)}(Q)$ (right panel) as a function of $(a Q)^{2}$. The lattice data at different quark masses are shown by open symbols. Fit curves for each quark mass and in the chiral limit are drawn. For $\Pi_{V+A}^{(0+1)}(Q)$ the full result in the chiral limit (dashed-dots curves are at the finite masses, and solid curve is in the chiral limit), as well as that without $\left\langle\alpha_{S} G^{2}\right\rangle / Q^{4}$ term (dashed curve), are shown.

but too large to make a reliable prediction for $b_{6}$ (squares). To quote the results we set $(a Q)_{\min }^{2}=$ 0.586 for both $\Pi_{V+A}^{(0+1)}(Q)$ and $\Pi_{V-A}^{(0+1)}(Q)$.

Figure 2 shows the lattice data for $\Pi_{V+A}^{(0+1)}(Q)$ (left) and $\Pi_{V-A}^{(0+1)}(Q)$ (right panel) at each quark mass and corresponding fit curves. In $Q^{6} \Pi_{V-A}^{(0+1)}(Q)$, the quark mass dependence is clearly observed. The main contribution comes from a dimension-six term $c_{6} m_{q} / Q^{6}$, while the dimension-four term $\langle m \bar{q} q\rangle / Q^{4}$ is sub-dominant $(\sim 20 \%)$, as its coefficient starts at $\mathscr{O}\left(\alpha_{s}\right)$. In the chiral limit, there is a small but non-zero value remaining in $\left.Q^{6} \Pi_{V-A}^{(0+1)}\right|_{\mathrm{OPE}}\left(Q^{2}\right)$ as shown by a dashed curve in the plot. This is due to the four-quark condensates $a_{6}$ and $b_{6}$.

The quark mass dependence of $\Pi_{V+A}^{(0+1)}(Q)$ is, on the other hand, not substantial as expected from the fit function (2.3). Our fit with the known value of $\langle\bar{q} q\rangle$ reproduces the data well. In the chiral limit, (2.3) is controlled by two parameters: $\Lambda \frac{(2)}{M S}$ and $\left\langle\left(\alpha_{s} / \pi\right) G G\right\rangle$ (apart from the constant term 
$c)$. The fit result in the chiral limit is drawn by a solid curve. The dashed curve, on the other hand, shows the result when the contribution from the $\left\langle\left(\alpha_{s} / \pi\right) G G\right\rangle$ term is subtracted. It indicates that the $Q^{2}$ dependence is mainly controlled by the perturbative piece while the dimension-four term gives a sub-dominant contribution. Numerically, we obtain $\Lambda \frac{(2)}{M S}=0.234(9) \mathrm{GeV}$ and $\left\langle\left(\alpha_{s} / \pi\right) G G\right\rangle$ $=-0.058(7) \mathrm{GeV}^{4}$. In order to estimate the systematic error due to the discretization effect, we calculate the vacuum polarization function at the one-loop level of lattice perturbation theory and extract the term of $\mathscr{O}\left((a Q)^{2}\right)$ out of the physical $\ln \left(Q^{2} / \mu^{2}\right)$ dependence of $C_{0}\left(Q^{2}, \mu^{2}\right)$. We then add this term to the fit function (2.3) and repeat the whole analysis, which yields $\Lambda \frac{(2)}{M S}=0.249(37) \mathrm{GeV}$ and $\left\langle\left(\alpha_{s} / \pi\right) G G\right\rangle=+0.11(15) \mathrm{GeV}^{4}$. We find that $\Lambda \frac{(2)}{M S}$ is not largely affected, while $\left\langle\left(\alpha_{s} / \pi\right) G G\right\rangle$ is very sensitive to the lattice artifact and in fact changes its sign. Other (Lorentz-violating) discretization effects due to $B_{n}^{J}$ and $C_{m n}^{J}$ are subtracted non-perturbatively so that the associated error should be negligible. (see ref.[5])

The truncation of the perturbative and operator product expansions is a possible source of the systematic error. In order to estimate the size of the former, we repeat the analysis using the fit formulae truncated at a lower order (two-loop level), and find that the change of $\Lambda \frac{(2)}{M S}$ is much less than one standard deviation. It indicates that the higher order effects are negligible. The error from the truncation of OPE is estimated by dropping the terms of $\mathscr{O}\left(1 / Q^{4}\right)$ from (2.3). From fits with higher $(a Q)_{\min }^{2}$ (between 0.79 and 0.89 ) to avoid contaminations of the $1 / Q^{4}$ effects, we obtain $\Lambda \frac{(2)}{M S}=0.247(3) \mathrm{GeV}$. The deviation of $\Lambda \frac{(2)}{M S}$ is about the same size as that due to discretization effect. The errors due to finite physical volume and the fixed topological charge in our simulation [13] are unimportant for the short-distance quantities considered in this work.

Our final result is

$$
\Lambda \frac{(2)}{M S}=0.234(9)\left(\begin{array}{c}
+16 \\
-0
\end{array}\right) \mathrm{GeV}
$$

where the first error is statistical and the second is systematic due to the discretization and truncation errors. The result is compatible with previous calculations of $\alpha_{s}$ in two-flavor QCD: $\Lambda \frac{(2)}{M S}$ $=0.250(16)(16) \mathrm{GeV}[14]$ and $0.249(16)(25) \mathrm{GeV}$ [15]. (The physical scale is normalized with an input $r_{0}=0.49 \mathrm{fm}$.) The four-quark condensate $a_{6}$ is obtained from $\Pi_{V-A}^{(0+1)}(Q)$ as $a_{6}(2 \mathrm{GeV})$ $=-0.0038(3)\left(\begin{array}{c}+16 \\ -0\end{array}\right) \mathrm{GeV}^{6}$, where the first error is statistical. The second error represents an uncertainty due to the truncation of the $1 / Q^{2}$ expansion. The central value is taken from the fit with $a_{8} / Q^{8}$ in (2.4) and the error reflects the shift when this term is discarded. The result agrees with the previous phenomenological estimates $-(0.003 \sim 0.009) \mathrm{GeV}^{6}[16]$. The other condensate is less stable; we obtain $b_{6}(2 \mathrm{GeV})=+0.0017(7) \mathrm{GeV}^{6}$ or $-0.0008(2) \mathrm{GeV}^{6}$ with or without the $\mathscr{O}\left(1 / Q^{8}\right)$ term, respectively.

\section{Summary}

With the exact chiral symmetry realized by the overlap fermion formulation, the analysis of the lattice data can be greatly simplified. For the case of the vacuum polarizations, the continuum form of OPE may be applied without suffering from additional operator mixings, such as the additive renormalization of the operator $\bar{q} q$. With an input for the chiral condensate from other sources, we can fit the lattice data at short distances and extract the strong coupling constant. The analysis does not require lattice perturbation theory, which is too complicated to carry out to the loop orders 
available in the continuum theory. Moreover we obtain the four-quark condensates $a_{6}$ and $b_{6}$, which are relevant to the analysis of kaon decays. An obvious extension of this work is the calculation in 2+1-flavor QCD, which is underway [17].

\section{Acknowledgments}

Numerical calculations are performed on IBM System Blue Gene Solution and Hitachi SR11000 at High Energy Accelerator Research Organization (KEK) under a support of its Large Scale Simulation Program (No. 08-05). This work is supported by the Grant-in-Aid of the Japanese Ministry of Education (No. 18340075, 18740167, 19540286, 19740121, 19740160, 20025010, 20340047, 20740156 ), and National Science Council of Taiwan (No. NSC96-2112-M-002-020MY3, NSC96-2112-M-001-017-MY3).

\section{References}

[1] S. L. Adler, Phys. Rev. D 10, 3714 (1974).

[2] L. R. Surguladze and M. A. Samuel, Phys. Rev. Lett. 66, 560 (1991) [Erratum-ibid. 66, 2416 (1991)]; S. G. Gorishnii, A. L. Kataev and S. A. Larin, Phys. Lett. B 259, 144 (1991).

[3] J. F. Donoghue and E. Golowich, Phys. Lett. B 478, 172 (2000); V. Cirigliano, E. Golowich and K. Maltman, Phys. Rev. D 68, 054013 (2003).

[4] J. Noaki et al. [JLQCD and TWQCD Collaborations], arXiv:0806.0894 [hep-lat].

[5] E. Shintani et al. [JLQCD Collaboration], arXiv:0807.0556 [hep-lat]; E. Shintani et al. [JLQCD Collaboration], PoS LAT2007, 134 (2007); E. Shintani et al. [JLQCD Collaboration], arXiv:0806.4222 [hep-lat].

[6] M. A. Shifman, A. I. Vainshtein and V. I. Zakharov, Nucl. Phys. B 147, 385 (1979).

[7] K. G. Chetyrkin, V. P. Spiridonov and S. G. Gorishnii, Phys. Lett. B 160, 149 (1985).

[8] G. Martinelli and C. T. Sachrajda, Nucl. Phys. B 478, 660 (1996).

[9] S. Aoki et al. [JLQCD Collaboration], arXiv:0803.3197 [hep-lat].

[10] H. Neuberger, Phys. Lett. B 417, 141 (1998); H. Neuberger, Phys. Lett. B 427, 353 (1998).

[11] H. Fukaya et al. [JLQCD Collaboration], Phys. Rev. Lett. 98, 172001 (2007).

[12] T. van Ritbergen, J. A. M. Vermaseren and S. A. Larin, Phys. Lett. B 400, 379 (1997).

[13] S. Aoki, H. Fukaya, S. Hashimoto and T. Onogi, Phys. Rev. D 76, 054508 (2007).

[14] M. Della Morte, R. Frezzotti, J. Heitger, J. Rolf, R. Sommer and U. Wolff [ALPHA Collaboration], Nucl. Phys. B 713, 378 (2005).

[15] M. Gockeler, R. Horsley, A. C. Irving, D. Pleiter, P. E. L. Rakow, G. Schierholz and H. Stuben, Phys. Rev. D 73, 014513 (2006).

[16] A. A. Almasy, K. Schicher and H. Spiesberger, and references therein.

[17] S. Hashimoto et al. [JLQCD collaboration], PoS LAT2007, 101 (2007). 